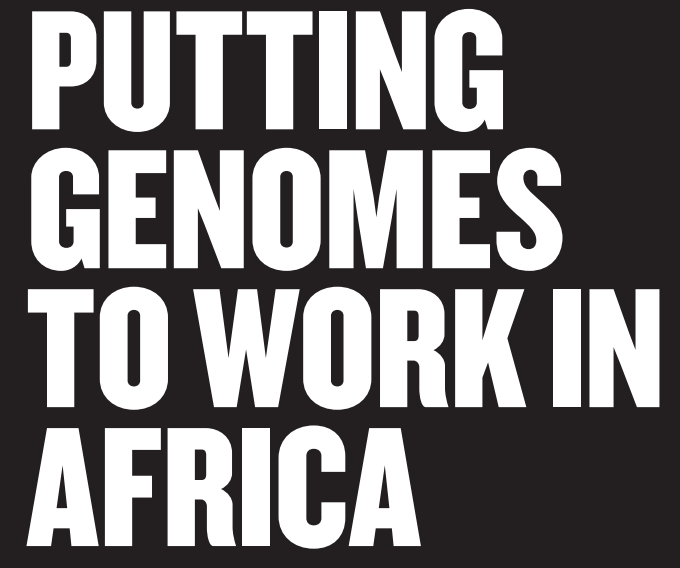

Investment promises to bring precision medicine to Africans. But will it help?

BY LINDA NORDLING
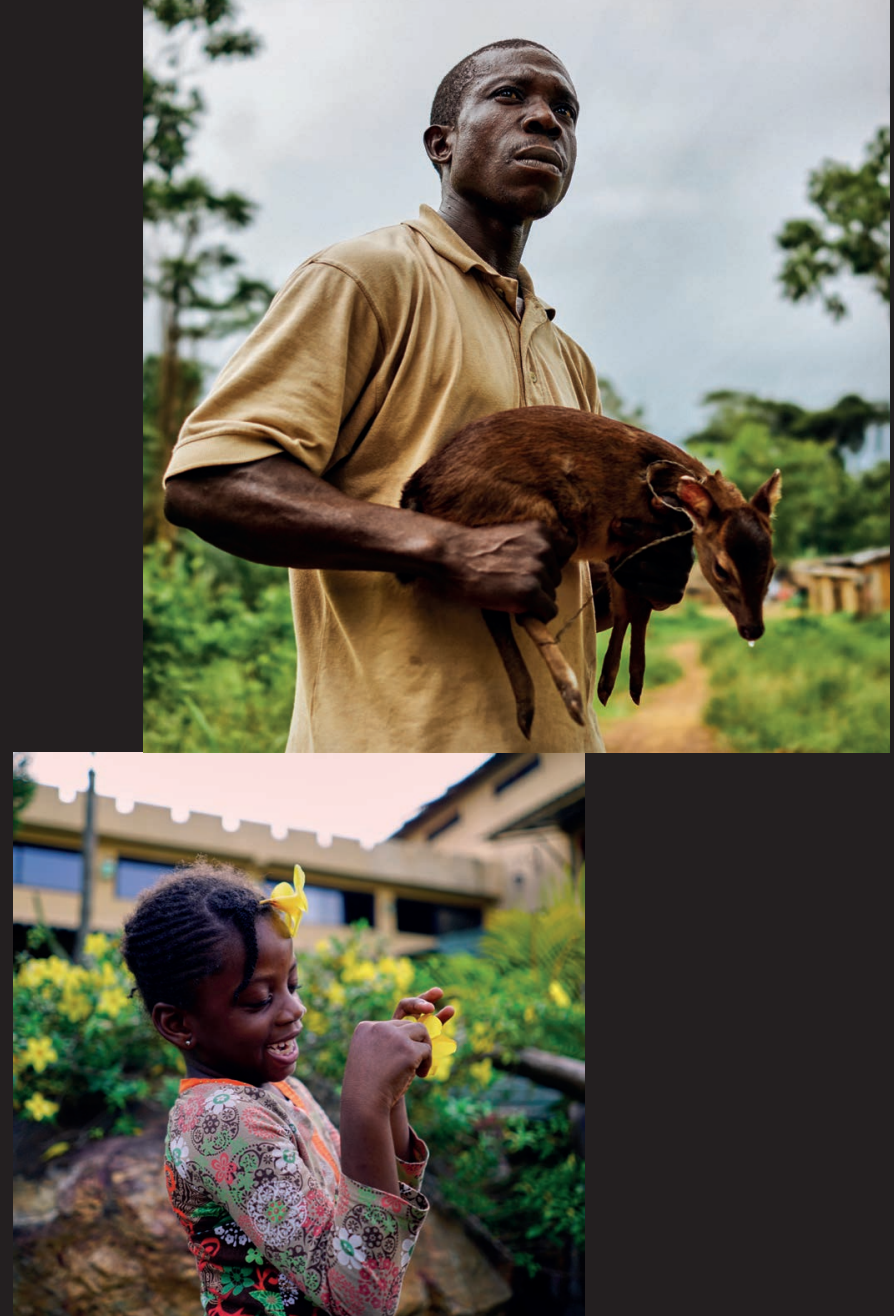

t took a public-health disaster for the Zimbabwean government to recognize the power of precision medicine. In 2015, the country switched from a standard three-drug cocktail for HIV to a singlepill combination therapy that was cheaper and easier for people to take every day. The new drug followed a World Health Organization recommendation to incorporate the antiretroviral drug efavirenz as a first-line therapy for public-health programmes. But as tens of thousands of Zimbabweans were put onto the drug, reports soon followed about people quitting it in droves.

Collen Masimirembwa, a geneticist and founding director of the African Institute of Biomedical Science and Technology in Harare, was not surprised. In 2007, he had shown that a gene variant carried by many Zimbabweans slows their ability to break down efavirenz ${ }^{1}$. For those with two copies of the variant - about $20 \%$ of the population - the drug accumulates in the bloodstream, leading to hallucinations, depression and suicidal tendencies. He had tried to communicate this to his government, but at the time efavirenz was not a staple of the country's HIV programme, and so the health ministry ignored his warnings.

Masimirembwa continued to publish his research, but the authorities took no heed until there was trouble. A lot of confusion could have been avoided if the government had listened, he says, "It's not a bad drug. We just know it can be improved in Africa."

Masimirembwa is a rare breed. Although scientists worldwide have been pushing for ways to improve health care by tailoring diagnostics and treatment to the environment, lifestyle and genes of individual patients, few researchers have taken this precision-medicine approach in Africa.

That may be changing. In the past five years, international researchfunding organizations have invested more than US\$100 million in projects to boost genetic research on people in Africa. These studies could lead to improved treatments for Africans as well as for people of recent African descent in Europe and the Americas, who tend to experience more ill health than other ethnicities - a situation that is often attributed to socioeconomic challenges, but which some scientists say could also have genetic roots.

Although few would question the importance of African genomics, opinions differ on whether this will translate into better care. Globally, precision medicine has failed to live up to its promise, even in countries that spend lots of money on health. And some argue that the money spent on investigating genes should instead be used to improve basic health care on the continent.

Many African scientists bristle at that simple calculus. They are frustrated that they have been left out of research on everything from health to human origins - a field that has particularly benefited from African genome data - and they want Africans to gain from the work. For Masimirembwa and others, the money presents an opportunity to take control of how genetic data are collected and used. "Unless capacity is built on the continent, Africans won't have a chance to participate," he says.

\section{POPULATION-SCALE PRECISION}

There's a big problem, however. Precision medicine is expensive. For a continent that, for the most part, struggles to provide even basic health care, tailor-made treatments for individual patients may seem like an unaffordable luxury.

Enter 'precision public health' - a new approach to precision medicine that bases health decisions on populations and communities rather than on individuals. It would use genomic insights into a country's population to inform general treatment programmes. For instance, a country might tweak its essential medicines list that specifies the drugs it buys in bulk at reduced rates from pharmaceutical companies, to avoid medicines that are known to cause problems in its population.

This is already happening in some places. Botswana - a middleincome country - stopped using the three-in-one drug containing efavirenz in 2016, opting instead for a newer and better-performing, but more expensive, drug called dolutegravir. The gene variant that causes problems with efavirenz is common in Botswana - around $13.5 \%$ of the population has two copies of it. And in 2015, Ethiopia banned the use of the painkiller codeine, because a high proportion of people in 


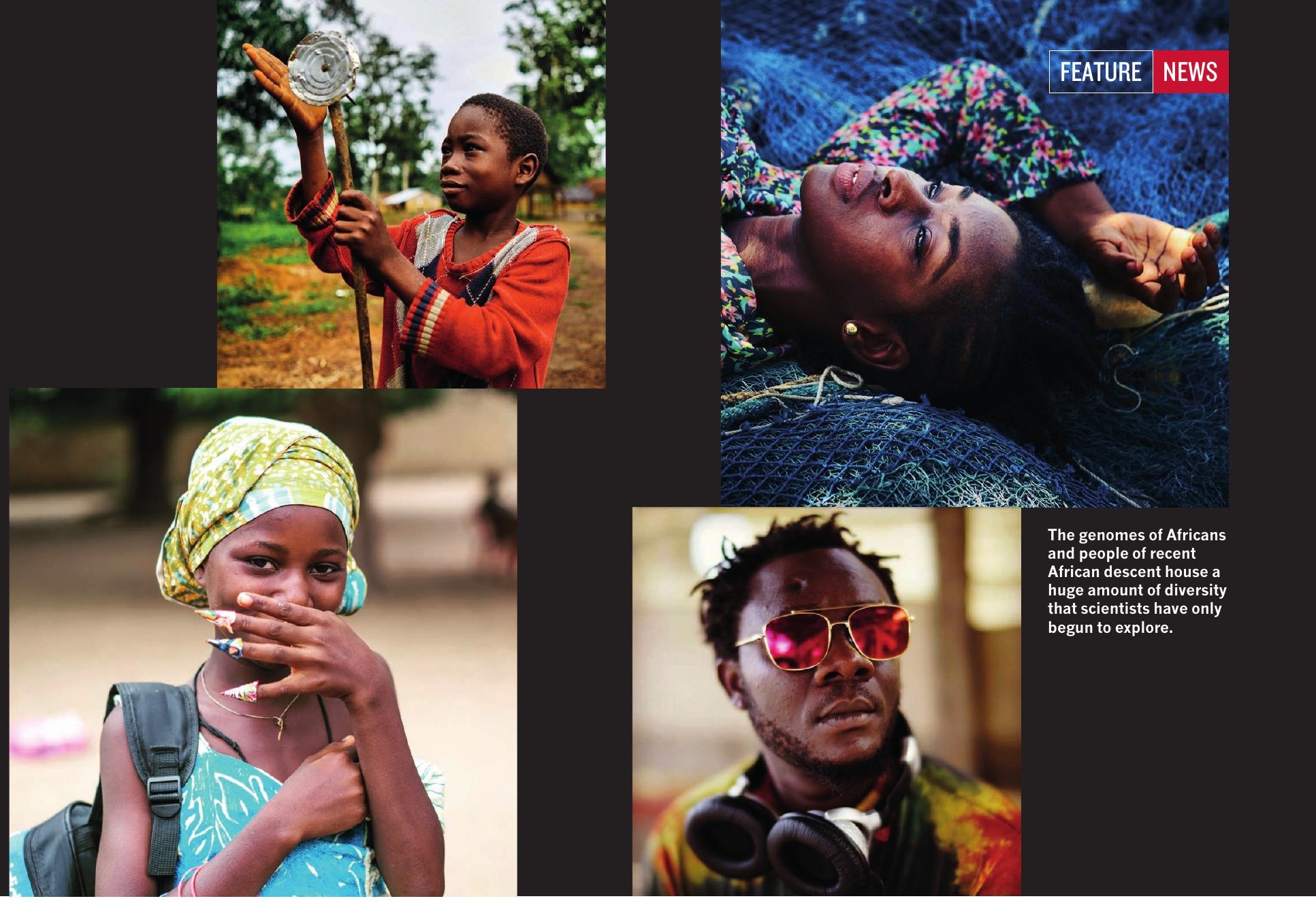

the country carry a gene variant that causes them to rapidly convert the drug into morphine, which can cause breathing problems or even death.

The precision public-health approach has great appeal for technology-savvy funding organizations that are eager to make a big impact on health. For instance, last October, the Bill \& Melinda Gates Foundation and the Alliance for Accelerating Excellence in Science in Africa (AESA), a funding platform based in Nairobi, Kenya, held a precision public-health summit in Ethiopia's capital Addis Ababa. The European Commission is drawing up plans for a precision public-health initiative. And AESA, which is supported by global funders and African organizations, also plans to expand into precision public health.

But to fulfil this vision, a lot of research needs to be done on African genomes. Most genomic studies so far have focused on white people of European descent. A meta-analysis published in Nature last year ${ }^{2}$ revealed that only $3 \%$ of global genome-wide association studies - which link genetic traits to patterns in health, disease or drug tolerance - had been performed on Africans, compared with $81 \%$ on people of European ancestry.

An added challenge is that Africans are the most genetically varied people on Earth. Africa is where humanity originated and where humans have lived the longest, so populations there have diverged more than on other continents. Its people have genetic variants that are found nowhere else.

These two factors mean that scientists are missing a big piece of the puzzle when it comes to human genetics, says Charles Rotimi, founding director of the National Institutes of Health's Center for Research on Genomics and Global Health in Bethesda, Maryland. Tests developed to inform treatment options for white people might be unsuitable for Africans and people of recent African descent. "We are in a position to make wrong diagnoses," he says.

Rotimi is one of the founders of the Human Heredity and Health in Africa (H3Africa) Initiative, created in 2010 by the London-based biomedical charity the Wellcome Trust and the US National Institutes of Health. Aiming to build genomics research capacity in Africa, the first round of the programme distributed $\$ 70$ million to African scientists who teamed up with partners from the United States and Europe (see 'An evolving consortium'). A second round, worth around \$64 million, is at the application stage.

The research targets conundrums that have dogged clinicians for some time - such as why Africans have a higher risk of developing chronic kidney disease, and do so at a younger age, than do white people. Nephrologist Dwomoa Adu at the University of Ghana Medical School in Accra, one of the principal investigators in the H3Africa Kidney Research Network, says there are no known environmental factors that explain this. But many Africans carry variants in the gene for apolipoprotein L1 (APOL1) that seem to confer an increased risk of developing kidney disease ${ }^{3}$. These variants have probably flourished in Africa because they confer resistance to trypanosomiasis, or sleeping sickness, a parasitic disease transmitted by the tsetse fly. But as life expectancy has increased in African countries, the incidence of kidney disease has risen markedly. And because there is little dialysis or kidney-transplant capacity on the continent, most people who develop the condition die, says Adu. "It's a nightmare illness."

Adu's study is testing the link between the APOL1 gene and kidney disease in Africa at a greater sensitivity than previous studies. But being able to predict the disease with a gene test will be of little use in places where treatment is inaccessible. So Adu is also looking to understand the mechanism by which the gene causes disease, in the hope that this will lead to new, more-affordable, treatments. "It might be possible to block the mechanism," he says.

Other H3Africa projects are looking for genetic clues to people's varying susceptibility to HIV progression, type 2 diabetes and stroke. One project is studying susceptibility to sleeping sickness. To find the genetic variations that might be causing this clinical diversity, $\mathrm{H} 3 \mathrm{Africa}$ has created a chip for quickly assessing variation in Africans. Such chips act as a tool for genome-wide association studies by giving researchers a catalogue of variants called single nucleotide polymorphisms (SNPs) that could be linked to risk for a particular disease or drug reaction. So far, H3Africa has identified 2.7 million previously unrecorded SNPs, 
and many have made it onto the chip. Samples from the San - a southern African indigenous group identified as the earliest genetic pool to split off from the rest of the human family tree - have a particularly rich vein of new SNPs to study. "We can't wait to explore them," says Nicola Mulder, a bioinformatician at the University of Cape Town in South Africa, who led work on the chip.

Although most of the H3Africa projects have yet to publish results, examples of the types of finding it might provide are starting to appear in the literature. For example, in March this year, Rotimi and his colleagues reported $^{4}$ that about $1 \%$ of West Africans, African Americans and others of recent African ancestry carry a gene variant that increases their risk of obesity. And a collaboration between South African and Italian scientists resulted, also last month, in the identification of a genetic variant ${ }^{5}$ that seems to increase the carrier's risk of heart disease and cardiac arrest. The researchers identified the variant by studying a South African family that has been hit hard by the disease, whose members did not carry any gene variants previously associated with the illness. Although it is not known how common the variant is in South Africa, it could play a part in the high levels of heart disease seen in the country.

These insights could lead to better treatment for Africans and people of recent African descent, and perhaps result in discoveries about human genetics. "We are all African beneath our skin, so understanding African genomes is going to be of global benefit," says Rotimi.

\section{COST AND CAPACITY}

The attention that genomics research is getting in Africa has not been without critics. Cost is a major concern. Like most developing regions, Africa is seeing a rapid rise in non-communicable diseases such as cancer. In developed countries, cancer treatments are profoundly informed by genomics. But many African nations have only a handful of cancer specialists, and limited capacity for diagnosis and treatment. Although breast-cancer rates, for example, are lower in parts of Africa than in developed countries, more Africans die from the disease and not just because of a lack of access to care - standard treatments sometimes seem less efficient in some African women. Still, basic cancer-therapy equipment may be higher on the wish list than new genomic tests tailored to African people's tumours. In April last year, for example, Uganda's only radiotherapy machine broke down, forcing people to travel to neighbouring Kenya for treatment, at their own cost.

There are those who think that projects such as H3Africa are overstating the significance of the genetic variance between Africans and Europeans, and its effects on treatment options. Reinhard Hiller, director of the Centre for Proteomic and Genomic Research, a non-profit bioinformatics organization in Cape Town, is pleased that there is growing interest in African genomics. But he thinks that many genomic approaches, especially for treating cancer, can be applied to Africans now. A biopsy from a black woman's breast tumour can undergo the same analysis as that of a European's to look for the tell-tale genetic signs of its origin, he argues. And starting to do this, even on a small scale, might provide more informative data than focusing only on the differences, he says. "We shouldn't try and prevent governments and societies in Africa from having access to cutting-edge solutions merely because they are deemed imperfect."

Insights from that could feed back to basic genomic research where outcomes warrant it. "We have to be a lot more pragmatic and do whatever we can do now. If we don't get on with it we'll be sitting here in $50-100$ years still without answers."

His lab is one of the few in Africa that can do genomic sequencing. At the moment, most of its therapeutic work is for the private health sector in South Africa. But he's hopeful that genomic medicine can make it into the public sector. The main constraint, besides the cost, is the lack of technicians and counsellors, he says - something that is also true in many wealthy countries, he adds.

But apart from the time it takes to do the research, the slow pace of government policy in Africa presents another stumbling block for the rollout of precision medicine. Masimirembwa's long-ignored

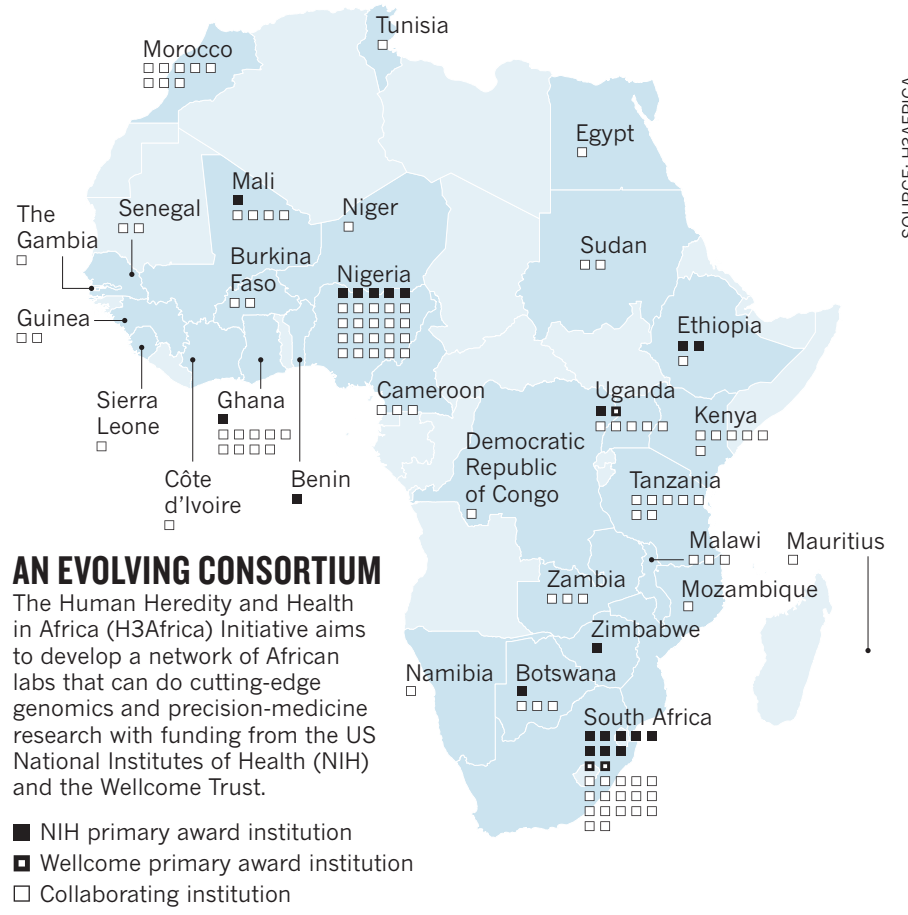

advice on efavirenz in Zimbabwe is a case in point.

As it turns out, the three-in-one HIV drug that the country rolled out in 2015 works well in people who tolerate it. But differentiating those individuals from the $20 \%$ or so who will probably have a bad reaction is difficult. Masimirembwa and his colleagues developed a genetic test for the gene variant that makes carriers sensitive to the drug. This, he says, could be used to identify people who need to be given a lower dose of efavirenz - something that he and his colleagues have determined decreases the risk of side effects while maintaining its efficacy. Last year he won a 500,000 rand $(\$ 39,000)$ commercialization grant from the South African government for his test. But he's up against the clock.

Zimbabwe's government, along with those in South Africa and Uganda, are considering going the same way as Botswana did, and ditching the efavirenz-based treatments entirely. Although the replacement drugs would not necessarily be any less effective, it would mean that Masimirembwa's test would no longer have a market — a disappointing fate for his discovery.

But Masimirembwa thinks that there is still time to make good on his idea. It takes governments years to make decisions on public health, he says, and the new drugs might be unaffordable. And while the Zimbabwean government mulls over options, many HIV-positive people in the country still face a difficult choice: take the drugs that are available and experience serious side effects, or stop taking them and risk developing AIDS. There are second-line alternatives, but most patients are told to 'hang in there' to see if the side effects subside, he says. Few are offered different drugs.

One good thing has come out of the debacle so far: it has opened the government's eyes to the value of Masimirembwa's research. In February this year he was awarded a $\$ 15,000$ national science award. "Whilst there was initially poor acceptance of our findings, the current national and regional support is very encouraging for the future of genomic medicine," he says. And if his test makes it from the bench to the bedside, it will set a good precedent, he adds. "We will have demonstrated that African scientists can take an idea from the lab to the market."

Linda Nordling is a freelance writer in Cape Town, South Africa. The Wellcome Trust sponsored travel associated with reporting of this story.

1. Nyakutira, C. et al. Eur. J. Clin. Pharmacol. 64, 357-365 (2008).

2. Popejoy, A. B. \& Fullerton, S. M. Nature 538, 161-164 (2016).

3. Parsa, A. et al. N. Engl. J. Med. 369, 2183-2196 (2013).

4. Chen, G. et al. Obesity 25, 794-800 (2017).

5. Mayosi, B. M. et al. Circ. Cardiovasc. Genet. 10, e001605 (2017). 